Voix et Images

\title{
Suzanne Lamy : le féminin au risque de la critique
}

\section{Sherry Simon}

Volume 13, numéro 1 (37), automne 1987

Suzanne Lamy

URI : https://id.erudit.org/iderudit/200684ar

DOI : https://doi.org/10.7202/200684ar

Aller au sommaire du numéro

Éditeur(s)

Université du Québec à Montréal

ISSN

0318-9201 (imprimé)

1705-933X (numérique)

Découvrir la revue

Citer cet article

Simon, S. (1987). Suzanne Lamy : le féminin au risque de la critique. Voix et Images, 13(1), 52-64. https://doi.org/10.7202/200684ar d'utilisation que vous pouvez consulter en ligne.

https://apropos.erudit.org/fr/usagers/politique-dutilisation/ 


\title{
Suzanne Lamy: le féminin au risque de la critique
}

\author{
par Sherry Simon, Université Concordia
}

Le féminisme est à la fois le dernier avatar du monde moderne et le symptôme de ce que nous appellerons ici, faute d'un meilleur terme, et à l'abri de son imprécision, le monde post-moderne. Il appartient très précisément à la crise du moderne.

Françoise Collin

Les femmes n'écrivent pas du tout au même endroit que les hommes. Quand elles n'écrivent pas dans le lieu du désir, elles n'écrivent pas, elles sont dans le plagiat.

Marguerite Duras

La parution de d'elles en 1979, en pleine période exubérante du féminisme, a donné naissance, au Québec, à ce que désormais on appellera la critique au féminin. Débordant d'un lyrisme enlevant, faisant preuve autant d'acuité que d'inventivité critique, d'elles proposait la jonction des mouvements intellectuels qui avaient le plus marqué la littérature des années 70: la pensée critique française (de Blanchot et de Barthes) et l'écriture des femmes. Rarement un si savant dosage aura produit résultat aussi percutant.

Au cours des années qui ont suivi d'elles, et jusqu'en 1985, Suzanne Lamy (S.L.) a consacré son abondant travail critique (essais, conférences, entretiens et comptes rendus critiques) presque exclusivement à l'écriture des femmes. Avec de plus en plus de finesse critique, avec une obstination exemplaire aussi, elle en fera le lieu d'un questionnement au centre des enjeux de la modemité.

En effet, l'analyse qu'elle fait de l'écriture des femmes se trouve liée aux problèmes critiques majeurs de la dernière décennie. Intervenant dans un contexte qu'elle définit comme post-structuraliste, très fortement attirée par la puissance de la lecture psychanalytique, S.L. se demandera à travers ses écrits si l'émergence d'un sujet féminin ne constitue pas, pour les études littéraires comme pour la théorie des discours en général, une interrogation capitale: l'articulation du sujet dans le langage et la réintégration du référent dans les théories textuelles; s'y trouvent posées aussi les questions de pouvoir et d'autorité dans le langage et dans les institutions. Comme chez Foucault, Blanchot et Barthes, le point de départ de toute lecture et de toute critique deviendra pour S.L. la question «Qui parle?». 
Et ces questions s'élaborent au moyen d'un discours critique renouvelé. Comme les Surréalistes, avec qui elle s'est d'abord confrontée aux mystères et aux plaisirs de l'hermétisme, S.L. n'oubliera jamais la matérialité du langage ni celle des autres, ni la sienne. Il y a écriture donc, mais écriture qui relève d'un discours critique. Si les essais de S.L. ressemblent de bien des façons à la «fiction-théorie» qu'elle s'applique à décrire, ils s'en distinguent par leur volonté de multiplier les points d'ancrage et les positions d'énonciation. Se déploient dans cette écriture à la fois le «je» du désir, le «nous» d'un mouvement social collectif et le «on» de la culture savante. C'est par cette plurivocité et surtout par le projet toujours renouvelé de définir l'écriture des femmes comme le lieu moderne de la rencontre du social et du littéraire que S.L. a ouvert un nouvel espace de discours.

\section{D'abord l'écriture, au féminin}

Le projet critique de S.L. prend sa source dans l'existence d'un nouvel objet d'analyse qu'elle appellera, à l'instar des écrivaines françaises, l'écriture au féminin. Les deux éléments de ce syntagme revêtent pour elle une égale importance. Le premier était facile à définir: il suffisait de renvoyer aux écrits de Roland Barthes; le second était nettement plus complexe. Chaque article, chaque conférence, reprendra la question du féminin dans un introït précautionnaire: d'abord une négation et ensuite une définition toute provisoire. Le féminin n'est pas une essence, il est l'expression d'une conjoncture sociale et linguistique. Il n'existe aucune nature de femme: De corps parlant, il n'y a pas (Quand je lis je m'invente $^{1}$, p. 12). Seulement les traces d'une culture millénaire et les effets de l'éclosion subite et violente d'une volonté féminine. Le féminin est l'expression d'expériences communes, d'un mode d'être dans la société, d'un désir commun d'inscrire dans l'écriture un entretemps vécu avec une singulière acuité, un rapport à l'écriture qui n'est pas étranger à la façon dont [les femmes] se perçoivent dans la société, le besoin de rompre avec des formes [...] traditionnelles dont l'adoption n'est jamais innocente (Quand, p. 32).

Si les présupposés théoriques du discours critique de S.L. restent extrêmement stables, le ton et les lieux de ses analyses changeront quand même quelque peu. Lié de très près à l'évolution des productions, très attentif à l'actualitế, ce discours sera inévitablement coloré par les changements du milieu. C'est ainsi que d'elles (1979), le produit d'une époque de découverte, crée un nouvel espace critique en explorant les interstices entre la parole et l'écriture. Quand je lis je m'invente (1984) fait preuve d'un raffinement critique où se sont atténuées quelque peu l'ardeur et la liberté du premier livre. Il s'attache davantage aux liens entre l'écriture des femmes et la modernité québécoise, et interroge avec insistance les conditions d'institutionnalisation et de réception des écrits de femmes.

1 Les références tirées de Quand je lis je m'invente seront dorénavant indiquées de la façon suivante: (Quand, p.). 
Dans son effort pour faire ressortir ce qui constituait à la fois la composante socio-historique et la qualité collective de l'écriture au féminin, S.L. ne fait pas souvent des lectures d'oeuvres particulières. Son objet habituel est un ensemble de textes, dont elle essaie de dégager les traits communs. On trouve tout de même quelques exceptions dans Féminité, subversion, écriture (1984) ainsi que dans certaines communications comme une analyse de l'Enregistrement de Monique La Rue et de la Constellation du Cygne de Yolande Villemaire. Les comptes rendus critiques, entretiens et éditoriaux écrits pour la revue Spirale sont soumis à la double exigeance de l'actualité et d'un traitement évaluatif.

Dans ses dernières communications, S.L. avait introduit une nouvelle problématique dans l'analyse des écrits des femmes. Elle questionnait le retour (symptomatique?) de certaines écrivaines vers les formes plus conventionnelles du récit et du roman, et mettait en cause de ce fait la «collusion» qui avait régné entre écriture au féminin et modernité au Québec et en France.

Malgré une certaine évolution donc dans la perspective et la relative diversité des lieux où il s'exprime (essais, comptes rendus, etc.), son travail de 1979 à 1985 fait preuve d'une cohérence interne très forte car il est porté par un projet très conscient de lui-même: bâtir un discours critique différent.

\section{Ensuite la critique, au féminin}

Contrairement à la règle qui veut que les approches critiques se définissent par leurs méthodes (structurales, psychanalytiques, marxistes, etc.), la critique au féminin de S.L. se définit d'abord par son corpus. Corpus relativement restreint puisque limité par la double frontière de l'actualité et de la modemite. (Dire qu'il s'agit d'écrivaines de langue française va de soi).

Pourquoi l'actualité? La préface à Féminité, subversion, écriture en fournit une justification: En leur temps, Christine de Pisan, Madame de Staël et George Sand ont sûrement été subversives. Mais, isolées par l'Histoire, ces écrivaines ne peuvent participer directement à la prise de conscience moderne des femmes ni à un débat féministe contemporain dont les éditrices de Remue-ménage et nousmêmes ressentons l'urgence (p. 6). L'accent sur l'actualité permet donc à S.L. de donner, en toute connaissance de cause, une part essentielle à la dimension sociale de l'écriture au féminin. Elle lui permet aussi d'en définir la part novatrice, aspect que son discours critique valorise fortement.

Et la modernité? Par écritures au féminin, je désigne les textes où il y a réellement écriture, au sens où il y a rapport du texte d̀ l'Histoire et aussi condensation dans le texte, présence de la forme qui rend possible une lecture plurielle et non univoque du texte (conférence inédite, Institut Simone de Beauvoir, p. 1). Il s'agit d'un parti pris critique, fondamental. En distinguant entre écrits féministes, littérature féminine, textes de femme ou il y a écriture (Quand, p. 12), S.L. déclare à multiples reprises que seule cette troisième catégorie saura la retenir. De Gabrielle Roy, de Marie-Claire Blais, d'auteurs 
masculins (à l'exception d'André Breton?) elle ne parlera guère. L'écriture constitue donc à la fois une valeur et un critère du choix du corpus.

On peut dire ainsi que son objet critique, le corpus qu'elle a choisi de traiter en exclusivité, trace une limite que ses explorations ne pourront pas dépasser et devient un encadrement à l'intérieur duquel son oeuvre reste prise. Il ne faut donc pas chercher dans l'oeuvre de S.L. une critique féministe telle qu'on peut la trouver chez certaines Américaines ou Anglo-Canadiennes, qui font de la critique féministe un outil de lecture et de révaluation de l'ensemble du corpus littéraire. (En fait S.L. ne semble même pas utiliser le terme «critique féministe», préférant «critique des écritures au féminin».) Il ne faut pas non plus y chercher le genre d'enquête historique que Christa Wolf a inauguré de façon si convaincante dans Cassandre 2 . En restreignant son corpus et en laissant largement de côté toute la dimension de l'histoire, S.L. était fidèle à l'esprit des années 70 . Aujourd'hui, peut-être, nous ressentons cette absence de manière plus aiguë. Sans perspective historique, l'éclosion subite de la nouveauté n'est-elle pas condamnée à une disparition aussi radicale?

Pour ce qui est des méthodes, c'est une voie pluraliste que S.L. propose pour la critique au féminin. Ce pluralisme serait fondé d'une part sur la priorité de «l'oeil au féminin» et d'autre part sur une utilisation judicieuse des leçons du marxisme, de la sociocritique, de la psychanalyse, du structuralisme ${ }^{3}:$ A prior rien n'empêche que l'oeil au féminin ne soit considéré comme un outil épistémologique aussi valable qu'un autre... seul cet oeil du féminin peut faire lever certains sens du texte, comme le rapport du texte à l'Histoire des femmes (Quand, p. 25). Mais il ne doit pas pour autant nous faire faire l'économie d'autres approches.

La critique pour S.L. comme pour Roland Barthes est une parole intermédiaire entre la science littéraire et la lecture ${ }^{4}$, un lieu de rencontre d'approches diverses, un lent et persistant travail de décryptage qui est fondé dans une éthique (pas d'esthétique sans éthique, elle le rappelle). C'est aussi un lieu où circule le désir. S.L. a su invoquer la plus grande variété de registres dans son

2 Le travail d'excavation d'un livre comme Cassandre de Christa Wolf (Alinéa, 1983, trad. A. Lance) relève d'une tout autre approche que celle empruntée par Suzanne Lamy. Cette dernière n'a-t-elle pas justement intitulé son essai sur des écrivaines du Québec: «Des enfants uniques, nés de père et de mère inconnus?»

3 Le contraste entre le consensus méthodologique qui règne dans l'écriture et la critique féministes au Québec (voir Caroline Bayard, «Qu'en est-il au fait de théorie depuis que les dieux sont morts?» dans Féminité, subversion, écriture) et la pluralité des méthodes, qui est cause de débat intense aux États-Unis, est assez saisissant. Pour un bon aperçu des enjeux methodologiques, voir les interviews avec Annette Kolodny, Carolyn Heilbruner, Kathleen Stimpson et Jane Gallop dans The New Orleans Revlew, vol. 13, no 4, Winter 1986. Les interviewers définissent la critique féministe américaine comme un des mouvements théoriques les plus éclectiques des années 80.

4 Roland Barthes, Critique et vérité, 1963, p. 63. 
écriture, mais le «rapport amoureux», la «recherche du plaisir» en sont souvent l'élément fondateur ${ }^{5}$. Les textes où le rapport affectif joue à plein (les essais d'elles, «Breton-Duras, B.D.») ont une force d'innovation tout particulière: ils ont fait irruption dans le discours critique québécois, le marquant d'une vivacité toute nouvelle.

Ce qui définit cependant sa démarche analytique, ce sont d'abord l'identification d'instances qui relient la parole et l'écriture, le social et le littéraire; et ensuite l'exploration tenace des multiples déterminations et significations du lieu d'énonciation. Ces deux dimensions définissent pour nous la contribution essentielle de S.L. à la constitution d'une critique au féminin.

\section{Entre la parole et l'écriture}

C'est par la voix que tout commence. Elle m'ont parlé, j'ai aimé leurs intonations, leurs pointillés (d'elles, p. 12). L'émergence du féminin se fait par la voix, par une parole située et partagée. S'il est question de voix autant que d'écritures, c'est que l'écriture des femmes semble garder trace de ce qui se passe dans la voix, comme si du corps à la lettre, un ton ou une fluidité palpable demeurait (Quand, p. 14). La parole exprime le rapport au réel, au pouvoir, pointe vers l'insertion sociale et l'immédiateté de l'expression féministe. C'est du côté de la voix donc que S.L. trouvera des formes qui diront la spécificité de l'expression des femmes. Le bavardage, le dialogue, la litanie, la polytonalité, devenues des catégories critiques, permettent de rendre compte à la fois de l'origine sociale de l'écriture et des formes mêmes que prendront leurs écritures. Ces formes, situées à la jonction de l'oral et de l'écrit, permettront d'explorer pourquoi le féminisme et l'écriture au féminin [sont] devenus à la fois fait social et fait de langage (p. 58).

\section{Le bavardage}

Le bavardage est «énonciation sans énoncé», production gratuite, lieu d'échange affectif entre femmes - une parole considérée dans ses fonctions de pure communication (ce que Jakobson nomme la fonction phatique et les austiniens la parole vue sous l'angle de la pragmatique). Le bavardage n'est pas une forme d'écriture, mais comme concept il permet d'effectuer une transvaluation des critères qui définissent l'efficacité du langage. Le bavardage est autant acte que parole, il est le lieu d'un dialogue où se joue notre rapport à l'autre, l'espace du déploiement du désir. Un jeu ou le je se déploie, prend plaisir dêtre ballotté par la marée montante. Les vagues s'entrelacent, naissent l'une de l'autre, chaque fois analogues, différentes. Terre et mer mêlées: une érotique (p. 30). Non plus absence, perte ou d'un hédonisme suspect, le bavardage devient un lieu de plaisir et d'ouverture: une fête et le moyen de régénération des

5 Voir les articles de Pierre Nepveu et de France Théoret dans le dossier consacré à l'oeuvre de Suzanne Lamy dans Spirale, été 1987, no 71. 
femmes, le lieu transitionnel où corps et idées, thèmes et désir se font champ d'expérience (p. 22).

Par de nombreux chemins, S.L. interroge cette parole, cette pratique proprement «in-finie», impossible à cemer dans sa totalité mais dont les liens avec l'histoire des femmes émergent sûrement. Tous les savoirs sont bons pour illuminer quelque aspect de son existence et les jugements portés sur lui: les dictionnaires et l'histoire étymologique de la langue, la philosophie, la littérature, la psychanalyse. Le commentaire vise non seulement à affirmer son existence en tant que pratique mais à révéler le rôle psychique, social, politique, du bavardage et sa fonction de moyen de révélation d soi-même (d'elles, p. 3233). Fondé dans une nouvelle éthique, le bavardage ne sera plus cette honte du langage décrite par Maurice Blanchot et qui, parole discréditée, entraîne dans le discrédit qui la frappe le jugement que l'on porte sur elle. Il sera libéré du jeu de pouvoir et de dénonciation qui le définissait jusque-là: Celui qui traite l'autre de bavard, se rend suspect d'un bavardage pire, prétentieux et autoritaire... on impose silence parce que, seul, on détient le droit de parler; on dénonce la parole vaine et on lui substitue la parole tranchante qui ne parle pas, mais qui commande 6 .

Cet essai sur le bavardage serait-il celui où s'expriment le mieux les jeux de connivence, la célébration, l'exaltation langagière de S.L.? Où se nouent de la manière la plus convaincante l'union du corps (social) et de la parole des femmes? Identifié à l'excès et à l'insignifiance de la parole, identifié donc à l'abus de la parole chez les femmes, le bavardage est une figure de la doxa qu'il fallait mettre à nu et dénoncer. Plus qu'un de ces mythes bourgeois dont Barthes a fait l'étude, c'est un paradigme proprement légendaire que S.L. a construit pour mieux le détruire.

\section{L'entretien, le dialogue}

L'entretien, le dialogue, ne sont pas plus des formes «d'écriture» que le bavardage. Mais l'analyse de S.L. montre que cette parole peut donner lieu à une écriture.

À partir d'une lecture des Parleuses de Marguerite Duras et Xavière Gauthier, elle définit la singularité de quelques entretiens de femmes par leur qualité d'échange véritable permettant l'évolution d'une pensée, et pas un «monologue déguisé» qui vise à faire valoir la pensée d'un des intervenants; et par leur désir de rendre compte d'un processus plutôt que de présenter un produit fini. La transcription brute de l'entretien vise à faire ressortir l'oralité et le caractère spontané des propos. Qu'est-ce que dialoguer, si ce n'est reconnaître son

6 «La Parole vaine», postface au Bavard de Loụis-René des Forêts, (10/18, 1963), p. 179. Voir aussi Gossip, Patricia Meyer Spacks, University of Chicago Press, Chicago and London, 1985. Il s'agit d'une lecture de certaines oeuvres de littérature anglaise à partir du concept de bavardage. 
impuissance d'atteindre seul à une parole fondatrice (p. 45)? C'est une parole toute de souplesse et de mouvements secrets (p. 46) où le rire, les complicités, le devenir, sont de la partie. C'est la forme d'une rencontre idéale où la pensée s'élabore, où il y a rigueur, franchise et découverte: et désir, où le pouvoir s'exerce le moins possible.

Mais l'entretien est plus qu'une parole, plus qu'une forme mineure d'expression. Il suggère le début d'une écriture: Une forme nouvelle est née là, du rythme de leurs respirations, de la contiguité faite de distance et d'harmonie, de la gravité légère de ces femmes pour qui écorce et épaisseur des êtres sont indissociables (d'elles, p. 51). On notera ainsi le soin que S. L. apporte à certains des entretiens qu'elle a fait paraître dans Spirale (avec notamment Luce Irigaray, Françoise Collin, Michèle Causse, Viviane Forrester, Chantal Chawaf, Marcelle Marini, Julia Bettinotti). Les meilleurs entretiens se distinguent justement par l'amorce d'un dialogue authentique où l'on sent le passage de réelles émotions et où le sujet de l'entretien touche au coeur de l'ecriture.

À cette fascination des rencontres entre femmes, évoquées dans l'essai sur le bavardage autant que dans l'étude sur le dialogue, elle reviendra en parlant de Marguerite Duras. Dans ses descriptions de la force de la personne et de la parole de Duras, elle fait comprendre la jouissance de l'idolâtrie en dépit des plus sains jugements critiques. Décrivant l'émotion suscitée par une rencontre collective avec Duras, elle écrit: Pourtant, loin d'être naïves, bien des personnes qui participaient à cette émotion savaient tout de la fonction de fétiche dévolue à l'artiste dans notre société, de la non-coïncidence entre le «je» écrivain... et le «je» présent dans la salle... («Duras s'avance vers nous», dans Duras à Montréal). Et pourtant... Dans un long entretien avec Duras, les moments de dialogue réel entrecoupent une conversation menée à partir d'une dissymétrie flagrante: Duras tutoie S.L., S.L. vouvoie Duras. Le dialogue est néanmoins la voie d'une recherche.

L'essai sur le dialogue, entrepris à partir de productions d'actualité (les dialogues féministes des couples Duras-Gauthier et Leclerc-Cardinal), est si suggestif qu'on ne peut que regretter que S.L. n'ait pas davantage exploré cette forme. (Une forme qu'elle utilise d'ailleurs elle-même dans deux essais de Quand je lis je m'invente). Il y aurait certainement lieu de mettre ces exemples contemporains en perspective historique, afin de tempérer des jugements peut-être trop facilement binaires (l'authentique versus la mauvaise foi, le processus versus le produit).

La litanie

Avec la litanie, caractéristique de l'écriture féministe des années 70, S.L. peut chevaucher résolument la parole et l'écriture, le collectif et l'individuel. Elle cherchera à lui donner les dimensions historiques et sociales les plus larges possible. $A$ la fois issue de la nuit des temps et des premières formes collectives de l'expression, la litanie rappelle toutes les formules infiniment recommencées, 
comme les jeux répétitifs des petites filles. Par sa nature de plainte, révélatrice de la soumission des femmes à l'ordre social et à la platitude des idées reçues, les rythmes de la litanie font penser aux chants de l'oppression, aux chants noirs, par exemple. (Pour souligner le rapport entre la problématique féministe et l'impuissance politique, elle aura recours au parallèle du colonisé. L'insertion de cette question dans le contexte québécois se fera à l'aide de références à Hubert Aquin, d'elles, p. 19, Quand, p. 51, etc.)

Mais par ce renversement de valeurs que l'on a vu déjà à l'oeuvre dans le bavardage et dans l'entretien, l'utilisation ludique et parodique de la litanie dans les écritures de femmes donne lieu à des expressions de révolte et de colère, à une internationale du langage, puisque du Québec, de France, d'Ttalie, de Belgique, du Portugal... les énoncés que nous entendons, sans être de même valeur, ont des airs de famille [...] (d'elles, p. 99). C'est comme si l'éclosion d'un langage nouveau ne pouvait être possible qu'une fois les vieilles formes maîtrisées et transformées. La litanie et son renversement parodique serait une forme intermédiaire, ritualisée, surexploitée peut-être (p. 99) mais caractéristique du mode d'entrée de bien des femmes dans une écriture assumée (p. 97).

Il y a donc un rapport privilégié entre la litanie et la parodie. Est-ce que cette présence importante ne placerait pas l'écriture des femmes près de l'esthétique du postmodeme plutôt que du modeme? Il y a eu peu d'effort jusqu'ici pour effectuer le rapprochement entre l'écriture au féminin et le postmoderne 7 et l'on peut certainement attribuer cette absence d'influence réciproque à l'évidence d'une différence radicale de projet. D'après Françoise Collin, c'est quand il se fait revendicateur que le féminisme se définit comme moderne; c'est lorsque, courtcircuitant la hiérarchie et l'opposition binaire des sexes, il installe partout la différence et l'hétérogénéité qu'il répond à l'appel du post-moderne ${ }^{8}$.

Pour S.L. c'est justement ce mélange de la revendication et du questionnement formel qui caractérise la litanie. Il s'agit d'une forme tout ambiguë,

7 Linda Hutcheon, «Shape shifters: Canadian women novelists and the challenge to traditions: it has become clear that contemporary literary theory is being pushed in new and important directions by feminist inquiry. Feminist theory has joined forces with post-structuralism to challenge and decenter such previously unassailable and solidly centered entities as continuous History and the rational Cartesian cogito [...] and to provide a critique of representation (p. 219). Mais: Janet Paterson (p. 315) cite Craig Owens: The absence of discussions of sexual difference in writings about postmodernism. as well as the fact that few women have engaged in the modernism/ postmodernism debate, suggest that postmodernism may be another masculine invention engineered to exclude women. «The Discourse of Others: Feminism and Postmodemism», in The Anti-Aesthetic: Essays on Postmodern Culture, ed. Hal Foster (Washington, Bay Press, 1983).

8 Françoise Collin, «le Féminisme et la crise du moderne», préface à Diane Lamoureux, Fragments et collages, Essai sur le féminisme québécois des années 70, Montréal, les éditions du Remue-ménage, 1986. 
témoignage de subordination et véhicule de promesses, proprement «troublante». Avec une perspicacité aigué, S.L. reconnaît que cette forme ne peut pas servir de salut esthétique. Tantôt régression au domaine des Mères (p. 79), tantôt progression vers un théâtre tout neuf (p. 91), la litanie ne donne lieu à rien de plus qu'à elle-même. Comment en serait-il autrement pour celle qui parle d'un lieu de discordance et de contradiction (p.97)?

\section{L'intertextualité}

La litanie est une forme possible de l'intertextualité. En s'arrêtant sur la polytonalité et l'intertextualité de trois textes au féminin de la modernité québécoise (Amantes de Nicole Brossard, Dieu de Carole Massé, «les Désordres du privé» de Louise Dupré, Quand p. 34-43), S.L. cherche à dégager les marques spécifiques qui font de ces écrits l'instance d'un phénomène général. Toute la valeur de la critique, répéte-t-elle, est là, dans cette conversion du singulier au collectif. Critique sage qui s'en tient à l'originalité de chacune de ces écritures. Elle a raison de chercher en quoi réside la singularité, mais elle se garde de souligner quelque analogie que ce soit d'un texte à l'autre. Et pourtant, si des traits communs n'avaient pas été reconnus, est-ce que le surréalisme, est-ce que la peinture automatiste... auraient accédé à l'existence (Quand, p. 41)? En l'absence d'un manifeste émergeant des écrivaines elles-mêmes, c'est à la critique de faire voir le front chaud qu'ils constituent et de souligner la relation évidente entre l'abondance de la citation, de l'intertextualité et l'histoire (p. 42).

Ainsi, le féminin dans l'écriture est-il toujours défini en rapport avec le collectif et le social. Face au risque pleinement assumé d'estomper les singularités, de produire un texte réducteur (Quand, p. 33), S.L. a proposé une critique résolument décidée à faire accéder à l'existence ce qui est un fait marquant de notre époque.

\section{Cette écriture, d'où l'interroger?}

La dimension sociale de sa critique ne s'arrête pas au constat du caractère collectif de l'écriture des femmes. Elle englobe la problématique des conditions d'émergence de cette écriture et l'analyse des conditions d'énonciation de la parole critique elle-même. Il faut bien considérer que le lieu d'où l'on parle compte souvent davantage dans nos sociétés que la parole elle-même (Quand, p. 101). L'ensemble de l'écriture de S.L. porte une attention extrême aux déterminants sociaux et idéologiques de la parole, une vigilance tirée des leçons de Barthes et de la sociocritique. Il n'est pas étonnant qu'elle ait placé cette interrogation au centre même de son projet critique?.

9 Edward Said définit la problématique du lieu en rapport particulièrement à la forme de l'essai: The central problematic of the essay as a form is its place, by which I mean a series of three ways the essay has of being the form critics take, and locate themselves in, to do their work. Place therefore involves 
La responsabilité critique exige qu'on prenne en considération les obstacles à la création chez les femmes. Parce que sans connivence avec le pouvoir, parce que longtemps restée loin des circuits publics, l'écriture des femmes ne peut voir le jour que si les conditions matérielles et idéologiques le permettent. ... ne parle que celle ou celui qui peut parler (d'elles, p. 51). Quelles femmes autrefois sont parvenues à écrire? Où? Comment? Avec quels moyens? (Quand, p. 13). Même aujourd'hui, comment écrire quand on a des enfants? Elle soulignera que Chantal Chawaf est mère de trois enfants (Spirale, no 24), elle parlera avec Marguerite Duras des enfants (Entretien, M.D. à Montréal). La prise de parole ne va pas de soi: S.L. sera sans pitié pour les universitaires qui utilisent d'une manière irresponsable le privilège de leur parole d'universitaire. (C'est un thème fréquent de ses chroniques dans Spirale).

L'écriture des femmes pour S.L. se définit en partie par le fait qu'elle parle de ses propres conditions de production et de ce fait constitue un dilemme pour la critique: comment la critique féministe doit-elle prendre en considération cette référence à ce qui est extérieur à l'écriture et à ce qui permet à l'écriture d'exister? Chez les femmes qui écrivent, si on admet en privé qu'il ne faut pas dire qu'un livre est bon parce qu'il a été écrit par une femme, le malaise n'en subsiste pas moins au moment de produire le texte critique. Mais faudrait-il vraiment que la solidarité entre femmes prenne le pas sur la lucidité et sur la connaissance? Est-il possible, chaque fois, de tenir compte de la situation d'ensemble des femmes par rapport aux institutions artistiques? Certes nous aimerions bien le faire. Dans ces conditions, peut-il exister une critique au féminin [...] Serait-ce là un travail de critique que celui qui évite l'évaluation explicite ou implicite? (Éditorial, Spirale, no 35 , juin 1983).

Les chroniques de S.L. dans Spirale essaient de concilier ces deux exigences: soutenir la parole au féminin et exiger d'elle la qualité esthétique de «l'écriture». Elle a donc dénoncé la fausse modernité et la misogynie des Fous de Bassan d'Anne Hébert (Spirale, no 29, novembre 1982), a adressé une sévère mise en garde contre la récupération du féminisme par des écrivaines comme Erica Jong (Spirale, no 17, mars 1981) et décelé des discordances au niveau de l'écriture dans Journal intime de Nicole Brossard (Spirale, no 44, juin 1984). Cette utilisation de la critique au sens évaluatif situe sa parole à la fois à l'intérieur et à l'extérieur du réseau des complicités. C'est seulement dans les pages de Spirale que S.L. prendra cette posture évaluative. On doit ainsi comprendre que l'essai, pour elle, n'était pas un lieu d'évaluation mais plutôt un lieu d'explication et de mise en rapport.

relations, affiliations, the critics fashion with the texts and audiences they address; it also involves the dynamic taking place in a critic's own text as it is produced. "The World, the Text and the Critic», dans le recueil du même titre, Harvard University Press, Cambridge Mass., 1983. 
Comprendre l'existence sociale de l'écriture et de la critique au féminin c'est aussi explorer les contraintes institutionnelles auxquelles elles participent. Certains essais font de S.L. une practicienne accomplie de la nouvelle critique institutionnelle au Québec. Ainsi a-t-elle écrit que L'approche esthétique a tout à gagner de la connaissance du contexte, des médiations avec le faire, le toucher, avec l'analyse de l'attente ou du refus du public (Quand, p. 53).

Constat: les écritures au féminin - telles qu'elles existent dans les revues, les lieux d'enseignement, le circuit des prix - vivent dans la marge au Québec 10 . Le but de la critique est donc de faire exister cette écriture en tant que telle et de l'intégrer aux institutions. Ces écritures, elles sont et seront peu de chose, si des encres rouges n'en dénudent le sens, si elles ne sont pas lues, commentées, intégrées a la tradition littéraire d̀ part entière (Quand, p. 20). C'est en réponse à cette nécessité qu'il faut interpréter les dialogues avec «Lui» que l'on retrouve dans Quand je lis je m'invente et qu'il faut aussi situer le travail actif de S.L. en tant que conférencière auprès des non-initiés.

Le désir de faire valoir une différence critique ne l'empêche pas cependant de suivre les codes universitaires et de faire soutenir sa parole par les références reconnues. Tous ses essais s'appuient sur une bonne batterie de notes infrapaginales. C'est une stratégie de validation mais c'est aussi une volonté d'afficher le lieu de sa parole critique. En désignant clairement le lieu d'où elles créent, écrivent, elles obligent ceux et celles qui vont commenter leurs choix, leurs oeuvres, leurs écrits à en faire autant, pour peu qu'il y ait désir de ne pas perdre tout crédit (Quand, p. 52). Autant elle exigeait des autres que ce lieu soit déclaré et pris en considération, autant elle tenait à rendre transparentes les sources et les visées de son écriture critique.

Ses propres essais critiques se conforment de manière fidèle à ces énoncés de principe, et surtout à celui-ci: féminisme et rigueur n'ont pas à être antithétiques (Quand, p. 23).

\section{L'emprise de la modernité}

Le principal contexte de l'écriture critique de S.L. est la modernité, concept et pratique. Écriture au féminin et modernité sont pour elle en étroite interdépendance et sont nées de conditions sociales et épistémologiques semblables. Les écritures au féminin seraient le greffon le plus vivace de la

10 Suzanne Lamy souligne à la fois la présence abondante des écrits de femmes dans l'institution et la précarité de leur existence dans la marge de cette institution. Elle souligne le peu d'attention qui leur a été accordée en tant que phénomène collectif. On entend un autre son de cloche de nos jours: dans la préface à l'impressionnant volume A Mazing Space: Writing Canadian Women Writing, les rédactrices affïrment: féminist criticism and writing have figured with increasing prominence here as elsewhere and have generated significant interaction with mainstream literature. On attend toujours les volumes qui engageront ce dialogue entre le féminisme et la littérature québécoise. 
modernité (Quand, p. 83), posant de façon inédite la question du sujet, rouvrant le débat théorique du rapport du texte au réel (p. 83), contestant le dogme de l'autonomie du texte.

C'est le but de ses lectures critiques de montrer justement que, jouant du double registre de l'écriture et du réel, les textes de femmes peuvent basculer dans les deux univers, peuvent parler doublement. Elle en fait la preuve dans sa lecture de la phrase Je suis un noeud de Nous parlerons comme on écrit de France Théoret (F, S, E). Le «je» qui parle est à la fois diffracté, morcelé, béance et voix de l'histoire des femmes. - L'effraction dans la langue est une affirmation, un acte critique quand elle met d̀ nu, dans le monde bardé d'interdits, le clivage entre le réel et les mots, entre les discours reçus et entre les langages, du trivial au plus savant (p. 144-45).

Mais pour combien de temps était-il encore nécessaire de faire la preuve du potentiel référentiel de l'écriture? En 1984 dans Spirale (no 40, mars 1984) S.L. avait salué le retour du référent dans le discours critique. C'est dire que ce gêneur, ce galeux presque d'où venait tant de mal - le référent pour tout dire - dont on s'était habilement débarrassé, retient à nouveau l'intérêt. Elle cite dans plusieurs lieux le texte d'Edouard Morot-Sir qui, contre le nihilisme de Blanchot ou de Baudrillard, voit le texte engagé dans un procès de référence et comprend le texte à critiquer comme une puissance de référence, dont il s'agit de déterminer l'intensité, la qualité et les modes 11 . C'est dire à quel point elle trouvait important de défendre le concept même de référence dans un climat critique qu'elle considérait réfractaire à cette notion.

Dans une de ses dernières communications (Sociêtés savantes, 1986), elle met en question la «collusion» dont elle avait elle-même déjà fait état entre l'écriture au féminin et la modernité. Elle suggère que l'emploi des formes conventionnelles du récit par certaines écrivaines remet de nouveau en question le rapport du texte au réel. Auteure elle-même d'un récit (la Convention, 1986), elle se demande si l'utilisation de formes plus classiques peut tout de même être porteuse de différence et d'innovation.

On peut voir à travers ces questionnements, je pense, les limites de certaines conceptions théoriques avec lesquelles S.L. avait travaillé, surtout après d'elles, et cela malgré le caractère très raffiné de ses analyses. D'un côté on trouve la modernité et l'écriture et de l'autre, le réel et l'histoire. Ce qui crée la spécificité d'une certaine écriture de femmes, c'est la possibilité de naviguer avec succès entre ces deux univers. Cette constatation, aussi utile qu'elle ait pu être comme mise en question de certaines conceptions bien à la mode, se révèle maintenant insuffisante.

11 Edouard Morot-Sir, «Texte, référence et déictique», dans Texte, no 1, 1982, p. 119. 
On peut bien croire que la distance prise par S.L. par rapport à la modernité - eût-elle pu poursuivre sa carrière de critique - se serait élargie pour donner lieu à une nouvelle configuration du rapport entre écriture des femmes et écriture tout court. Est-ce que le nouvel intérêt pour l'histoire et le débat autour du postmoderne auraient modifié les prémisses d'une critique dominée par l'exigence du moderne? Nous ne pouvons que rêver aux voies que sa carrière aurait pu suivre.

Le travail critique de Suzanne Lamy aura agi dans le monde: il a accompli pour l'écriture des femmes un indiscutable travail d'autorisation. Fruit d'une mission singulière, modèle d'imagination et de rigueur, il est maintenant le point de départ de toute critique au féminin devant advenir au Québec. Pourra-t-elle servir aussi à l'instauration d'une nouvelle critique qui se définira comme féministe, et dont l'espace de déploiement sera infini?

\section{Ouvrages de référence}

d'elles, Montréal, l'Hexagone, 1979.

Quand je lis je m'invente, Montréal, l'Hexagone, 1984.

Féminité, Subversion, Écriture, Textes réunis et présentés par Suzanne Lamy et Irène Pagès, Montréal, Remue-ménage, 1983.

Marguerite Duras à Montréal, Textes réunis et présentés par Suzanne Lamy et André Roy, Montréal, éditions Spirale, 1981.

«Des ambiguïtés de la fiction à la tentation du récit», présenté à l'APFFUC, Sociétés savantes, juin 1986. Repris en substance dans sa contribution à Tessera, 1987.

"Grandeurs et misères de la critique au féminin", communication donnée à l'Institut Simone de Beauvoir, Université Concordia, 8 février 1984.

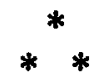




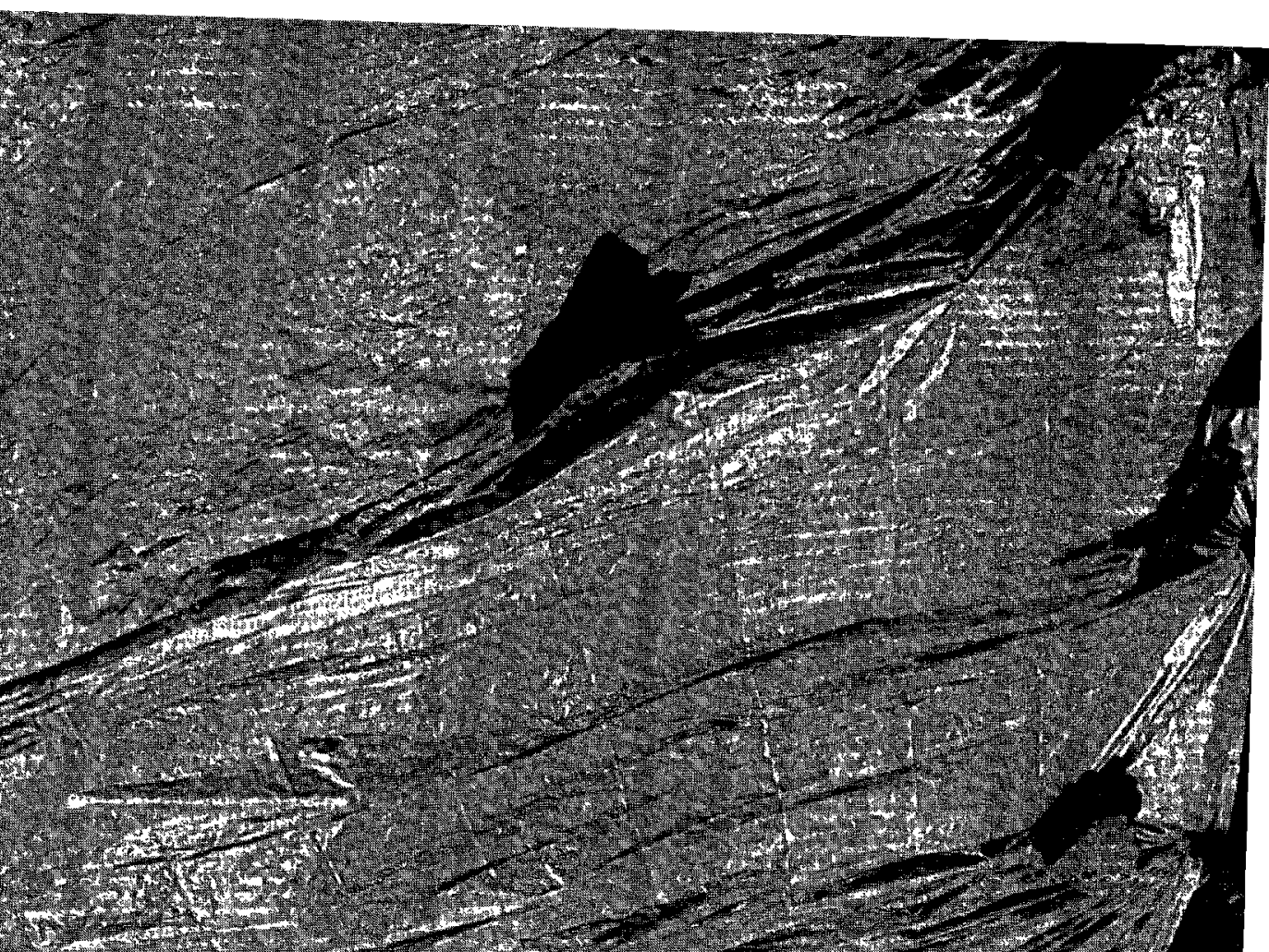

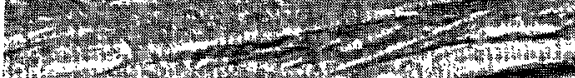

Whin

1
$y$

1
$y$

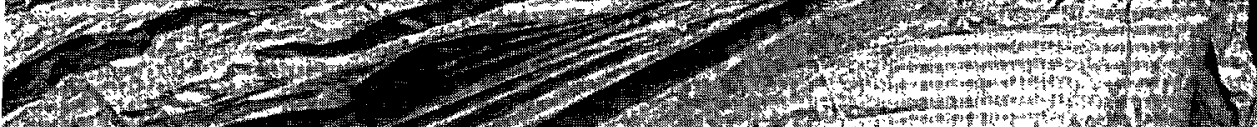

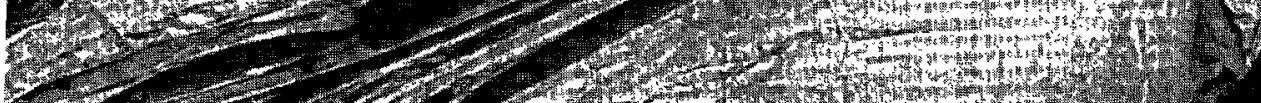

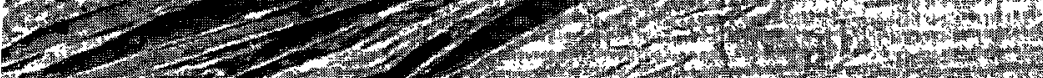

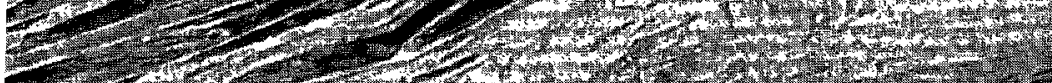

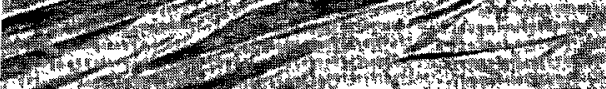

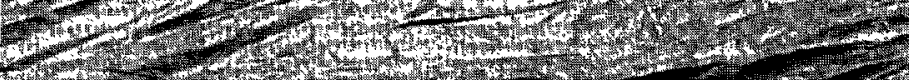

H.

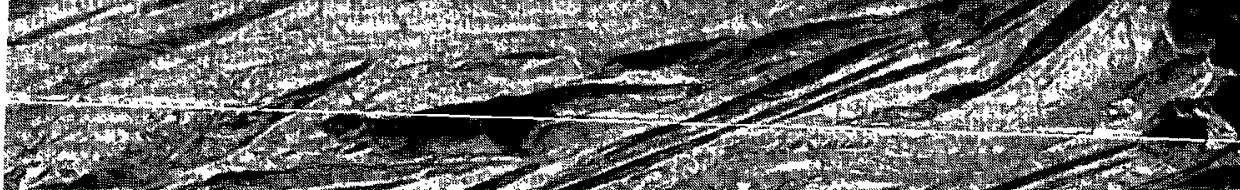

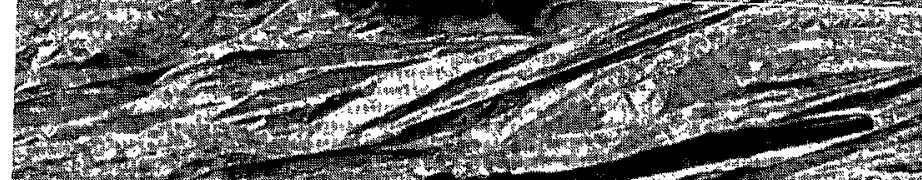

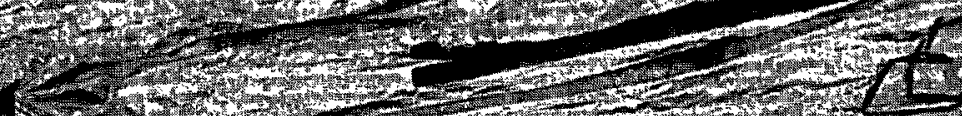

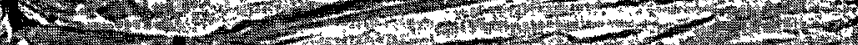

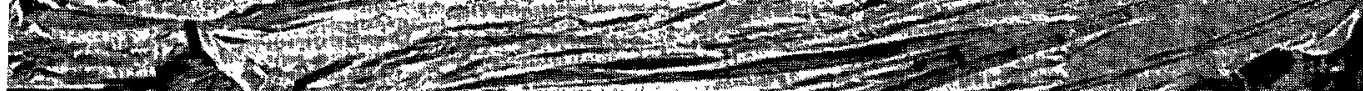

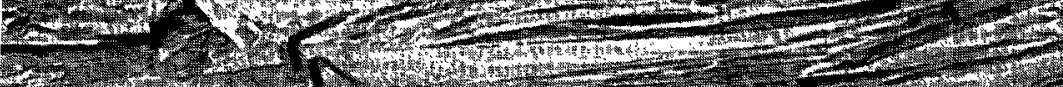

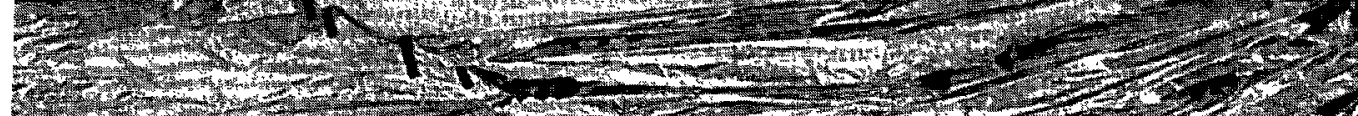

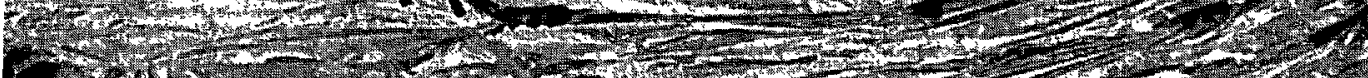

